\title{
Characterization of the Cofactors Involved in Nonenzymatic Metmyoglobin/Methemoglobin Reduction In Vitro
}

\author{
M. Denzer ${ }^{1}$, C. Mowery ${ }^{1}$, H. Comstock ${ }^{1}$, N. B. Maheswarappa ${ }^{2}$, G. G. Mafi ${ }^{1}$, \\ D. L. VanOverebeke ${ }^{1}$, and R. Ramanathan ${ }^{1 *}$ \\ ${ }^{1}$ Department of Animal and Food Sciences, Oklahoma State University, Stillwater, OK 74078, USA \\ ${ }^{2}$ National Research Centre on Meat, Chengicherla, AP 500039, India \\ *Corresponding author. Email: ranjith.ramanathan@okstate.edu (R. Ramanathan)
}

\begin{abstract}
Previous research reported the role of nonenzymatic metmyoglobin (MetMb) and methemoglobin (MetHb) reduction in meat color; however, limited studies have characterized the cofactors involved in nonenzymatic reduction. The objective of this study was to characterize electron donors and carriers in nonenzymatic MetMb and MetHb reduction at various temperatures and postmortem muscle $\mathrm{pHs}$ in vitro. Methylene blue and cytochrome $c$ (cyt-c) were evaluated as electron carriers and nicotinamide adenine dinucleotide, reduced form (NADH) and ascorbate were considered as electron donors. All combinations of electron donors and carriers were evaluated in the following order: NADH plus methylene blue, ascorbate plus methylene blue, NADH plus cyt-c, and ascorbate plus cyt-c. Spectrophotometry was utilized to monitor the rates of reduction. The results indicated that methylene blue was an effective electron carrier than cyt-c in the presence of $\mathrm{NADH}$. Temperature and $\mathrm{pH}$ had cofactor-specific effects on nonenzymatic MetMb and MetHb reduction. Lower temperature resulted in an increased nonenzymatic MetMb reduction for methylene blue regardless of electron donor (ascorbate, $P=0.03$, NADH, $P=0.04$ ). As pH increased, MetHb reduction was enhanced in the presence of ascorbate plus cyt-c. Nonenzymatic MetHb reduction was numerically lower than nonenzymatic MetMb reduction in the presence of NADH plus methylene blue. In summary, in addition to NADH, the current in vitro research demonstrated that ascorbate plus cyt-c could contribute to nonenzymatic MetMb and MetHb reduction at meat-pH and storage temperature.
\end{abstract}

Key words: metmyoglobin reduction, methemoglobin reduction, myoglobin, hemoglobin, meat color

Meat and Muscle Biology 4(1): 7, 1-10 (2020)

doi: $10.22175 / \mathrm{mmb} .9507$

Submitted 22 September $2019 \quad$ Accepted 23 November 2019

\section{Introduction}

Myoglobin and hemoglobin are the primary proteins responsible for meat color. Myoglobin, a monomer, contains a globin and one heme, whereas hemoglobin is a tetramer with 4 polypeptide chains, each containing one heme (Kendrew et al., 1960; Perutz et al., 1960). Meat color is influenced by the redox state of the heme iron of myoglobin and the ligand bond to the sixth position of myoglobin. Autoxidation of hemoglobin and myoglobin results in the formation of met-heme forms (Richards et al., 2002; Grunwald and Richards, 2006; Yin et al., 2017) and brown appearance on the lean surface (Carpenter et al., 2001; AMSA, 2012). Physiologically, tissue has an inherent ability to limit the levels of metmyoglobin (MetMb) and methemoglobin (MetHb) by enzymatic or nonenzymatic pathways (Brown and Snyder, 1969; Arihara et al., 1995). Nicotinamide adenine dinucleotide, reduced form $(\mathrm{NADH})$ is an important electron donor involved in MetHb or MetMb reduction (Faustman and Cassens, 1990; Tang et al., 2005; Ramanathan and Mancini, 2018). However, limited knowledge is currently available on the roles of other electron carriers and electron donors involved in MetHb and MetMb reduction at postmortem muscle conditions. 
NADH is an important reducing equivalent responsible for MetMb reduction and color stability. For example, improved color stability of lactate-enhanced loins can be attributed to increased NADH formation by lactate dehydrogenase activity. Further, NADH formed can be utilized for various MetMb reduction pathways, including nonenzymatic MetMb reduction (Kim et al., 2006). The concentration of NADH is related to color stability (Liu et al., 2014). Wet-aged beef steaks had $29 \mathrm{nmol}$ of NADH per $\mathrm{kg}$ of muscle tissue on day 3 of aging, and on day 28 of aging, NADH concentration decreased to $12 \mathrm{nmol}$ of NADH per kg of muscle tissue (Mitacek et al., 2019).

In nonenzymatic MetMb and MetHb reduction, an artificial electron carrier moves an electron from NADH to MetMb (Brown and Snyder, 1969), whereas in the enzymatic method, an enzyme such as NADHdependent cytochrome $b_{5}$ reductase transfers an electron from NADH to MetMb via an electron carrier (Arihara et al., 1995). Various cofactors such as methylene blue and cytochrome $c$ (cyt-c) have been reported to be involved in MetMb or MetHb reduction. Nonenzymatic reduction of MetMb by NADH in the presence of ethylenediaminetetraacetic acid (EDTA) and methylene blue in vitro is reported to be as efficient as the enzymatic reduction (Brown and Snyder, 1969). Pascal and Tarbell (1957) reported that EDTA could prevent metal catalysis and also serve as a chelating agent. Elroy et al. (2015) studied the nonenzymatic reduction of bovine, equine, and porcine MetMb in the presence of NADH, EDTA, and methylene blue. Unlike MetMb reduction, the MetHb reduction is mainly reported in erythrocytes (Beutler and Baluda, 1963). The reduction of MetHb by ascorbic acid in human erythrocytes has also been well established (Tomoda et al., 1978a). The time course of MetHb reduction by ascorbic acid was studied by Tomoda et al. (1978b), and these researchers concluded that various intermediates are involved in MetHb reduction. More studies are needed to characterize electron carriers and donors involved in MetHb reduction pathways in muscle foods.

Methylene blue acts as an artificial electron carrier to reduce MetMb and MetHb. In addition, methylene blue has the ability to accept and donate electrons readily cycling from oxidized to reduced states (Atamna et al., 2012). Previous research has indicated that cyt-c acts as an electron carrier for electron movement from the outer mitochondrial membrane to the inner mitochondrial membrane (Bernardi and Azzone, 1981). Exogenous ferricytochrome $c$ has the ability to donate electrons to exogenous NADH of the mitochondria (Lofrumento et al., 1991). The use of the ascorbic acid and cyt-c system for nonenzymatic $\mathrm{MetMb}$ or MetHb reduction has not been reported at meat $\mathrm{pH}$ and storage conditions. Therefore, the objective of the present study was to determine the role of various electron donors and cofactors on nonenzymatic $\mathrm{MetMb}$ and $\mathrm{MetHb}$ reduction at different $\mathrm{pH}$ and temperature conditions in vitro.

\section{Materials and Methods}

\section{Materials and chemicals}

Equine skeletal myoglobin, hemoglobin from bovine blood, methylene blue, potassium phosphate monobasic $\left(\mathrm{KH}_{2} \mathrm{PO}_{4}\right)$, potassium phosphate dibasic $\left(\mathrm{K}_{2} \mathrm{HPO}_{4}\right)$, sodium L-ascorbate, EDTA disodium salt dihydrate, cyt-c from bovine heart, and $\beta-\mathrm{NADH}-$ reduced dipotassium salt were purchased from Sigma Chemical Company (St. Louis, MO). For the preparation of Drabkin's solution, potassium cyanide (KCN), potassium ferricyanide $\left(\mathrm{K}_{3} \mathrm{Fe}(\mathrm{CN})_{6}\right)$, and sodium bicarbonate $\left(\mathrm{NaHCO}_{3}\right)$ were purchased from Sigma Chemical Company (St. Louis, MO). All chemicals were of reagent grade or greater purity.

\section{Hemoglobin and myoglobin preparation}

Equine MetMb has been previously used in numerous studies to determine the characteristics of meat color. Bovine MetMb shares $88.2 \%$ of amino acid sequence with equine MetMb (www.expasy.org), and bovine MetMb is not commercially available in freezedried form. Therefore, lyophilized equine MetMb was compared with lyophilized bovine MetHb.

The concentration of bovine MetHb was determined by adding MetHb solution in Drabkin's solution to form a stable cyanmethemoglobin, and the hemoglobin concentration was determined by measuring absorbance at $540 \mathrm{~nm}$. The concentration of MetHb was adjusted using Drabkin's solution to achieve a concentration of $0.08 \mathrm{mM}$ (assuming an extinction coefficient of $11.0 \mathrm{mM}^{-1} \mathrm{~cm}^{-1}$ ) (Zijlstra and Buursma, 1997). The extinction coefficient was previously determined using one heme group and one globin moiety of cyanmethemoglobin (Zijlstra and Buursma, 1997). Drabkin's solution was not used for the preparation of equine MetMb. Equine MetMb was dissolved in phosphate buffer, and MetMb concentration was confirmed by measuring the absorbance at $525 \mathrm{~nm}$ to achieve a final concentration of $0.08 \mathrm{mM}$ 
(assuming an extinction coefficient of $7.6 \mathrm{mM}^{-1} \mathrm{~cm}^{-1}$ ) (Broumand et al., 1958).

\section{Nonenzymatic MetMb and MetHb reduction}

The methodology used by Brown and Snyder (1969) was modified to determine the characteristics of the cofactors involved in nonenzymatic MetMb and MetHb reduction in vitro. The assay mixture contained varying combinations of NADH $(0.71 \mathrm{mM})$, ascorbate $(2$ or $5 \mathrm{mM})$, methylene blue $(0.025 \mathrm{mM})$, cyt-c $(0.094 \mathrm{mM})$, and EDTA $(0.71 \mathrm{mM})$ with the equine MetMb or bovine MetHb $(0.08 \mathrm{mM})$ in phosphate buffer $(50 \mathrm{mM})$ in a clear 96 -well plate. As components were excluded in the assay, phosphate buffer $(50 \mathrm{mM})$ at specified $\mathrm{pH}$ replaced the specified volume of the removed substrate to maintain the same assay volume across all experiments. To initiate the reduction reaction, NADH $(0.71 \mathrm{mM})$ or ascorbate ( 2 or $5 \mathrm{mM}$ ) were added last to the assay mixture. The same concentration of NADH and ascorbate were considered for their reducing abilities. However, preliminary research indicated that $0.71 \mathrm{mM}$ of ascorbate was not effective in reducing MetMb. Therefore, a greater concentration of ascorbate was required for reduction with cyt-c compared with ascorbate combined with methylene blue. At $2 \mathrm{mM}$, ascorbate was effective in reducing MetMb in the presence of methylene blue but not in the presence of cyt-c. Therefore, increasing the concentration of ascorbate to $5 \mathrm{mM}$ in the presence of cyt-c resulted in the more effective reduction of MetMb.

The absorbances at 582 and $576 \mathrm{~nm}$ were used to measure the MetMb and MetHb reduction, respectively, with the kinetics option in a Molecular Devices SpectraMax M3 Multi-mode microplate reader (San Jose, CA). Nonenzymatic MetMb reduction was calculated as nanomoles of MetMb reduced per minute during the linear phase of the reaction using a difference in molar absorptivity of $12,000 \mathrm{~mol}^{-1} \mathrm{~cm}^{-1}$ at $582 \mathrm{~nm}$. Similarly, nonenzymatic MetHb reduction was calculated during the linear phase of the reaction as nanomoles of MetHb reduced per minute using a difference in molar absorptivity of $15,190 \mathrm{~mol}^{-1} \mathrm{~cm}^{-1}$ at $576 \mathrm{~nm}$. The selected wavelengths have been established as the maxima for oxymyoglobin and oxyhemoglobin (Bowen, 1949; Zijlstra and Buursma, 1997). Three separate experiments were carried out to determine the effects of cofactors, $\mathrm{pH}$, and temperature on nonenzymatic MetMb and MetHb reduction.

\section{Experiment 1: Effects of cofactors}

The assay mixture contained varying combinations of cofactors with the equine MetMb or bovine MetHb $(0.08 \mathrm{mM})$ in phosphate buffer $(50 \mathrm{mM})$ at $\mathrm{pH} 5.6$ and $25^{\circ} \mathrm{C}$ (Table 1). For all treatments, nonenzymatic $\mathrm{MetMb}$ and MetHb reduction were calculated during the linear phase of the reaction for $10 \mathrm{~min}$. Based on the preliminary studies, some of the cofactor combinations required more time for the reduction; therefore, the reaction time was extended to $25 \mathrm{~min}$.

\section{Experiment 2: Evaluation of the temperature effect on cofactors}

The treatment combinations utilized in experiment 1 were combined in the assay mixture to measure the effect of temperature at 4 and $25^{\circ} \mathrm{C}$ on cofactors at pH 5.6 (Table 2). As previously mentioned, a greater concentration of ascorbate was required for reduction in the presence of cyt-c. For temperature consistency at $4{ }^{\circ} \mathrm{C}$, the reactants were kept on ice, and the well plate was kept on ice in an enclosed cooler during the reaction period. The absorbance was measured at 5 -min intervals using the endpoint set at $582 \mathrm{~nm}$ for oxymyoglobin and $576 \mathrm{~nm}$ for oxyhemoglobin on the Molecular Devices SpectraMax M3 Multi-mode

Table 1. Treatment combinations added to equine metmyoglobin $(0.08 \mathrm{mM})$ and bovine methemoglobin $(0.08 \mathrm{mM})$ to measure nonenzymatic metmyoglobin and methemoglobin reduction for experiment 1

\begin{tabular}{|c|c|c|c|c|c|c|}
\hline \multicolumn{7}{|c|}{ Substrates $^{\mathrm{a}}$} \\
\hline & Substrates combination & $\begin{array}{c}\text { NADH } \\
(0.71 \mathrm{mM})\end{array}$ & $\begin{array}{l}\text { Ascorbate } \\
(5 \mathrm{mM})\end{array}$ & $\begin{array}{c}\text { Methylene blue } \\
(0.025 \mathrm{mM})\end{array}$ & $\begin{array}{c}\text { Cytochrome } c \\
(0.094 \mathrm{mM})\end{array}$ & $\begin{array}{c}\text { EDTA } \\
(0.71 \mathrm{mM})\end{array}$ \\
\hline 1 & NADH & + & - & - & - & - \\
\hline 2 & $\mathrm{NADH}+$ methylene blue & + & - & + & - & - \\
\hline 3 & $\mathrm{NADH}+$ methylene blue + EDTA & + & - & + & - & + \\
\hline 4 & $\mathrm{NADH}+$ cytochrome $c+$ EDTA & + & - & - & + & + \\
\hline 5 & Ascorbate + cytochrome $c+$ EDTA & - & + & - & + & + \\
\hline
\end{tabular}

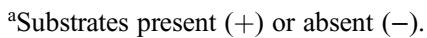

$\mathrm{EDTA}=$ ethylenediaminetetraacetic acid; $\mathrm{NADH}=$ nicotinamide adenine dinucleotide, reduced form. 
Table 2. Treatment combinations added to equine metmyoglobin $(0.08 \mathrm{mM})$ and bovine hemoglobin $(0.08 \mathrm{mM})$ to measure nonenzymatic metmyoglobin and methemoglobin reduction for experiment 2 and 3

\begin{tabular}{|c|c|c|c|c|c|c|c|}
\hline \multicolumn{8}{|c|}{ Substrates $^{\mathrm{a}}$} \\
\hline & Substrates combinations & $\begin{array}{c}\text { NADH } \\
(0.71 \mathrm{mM})\end{array}$ & $\begin{array}{l}\text { Ascorbate } \\
(2 \mathrm{mM})\end{array}$ & $\begin{array}{c}\text { Ascorbate } \\
(5 \mathrm{mM})\end{array}$ & $\begin{array}{c}\text { Methylene blue } \\
(0.025 \mathrm{mM})\end{array}$ & $\begin{array}{c}\text { Cytochrome } c \\
(0.094 \mathrm{mM})\end{array}$ & $\begin{array}{c}\text { EDTA } \\
(0.71 \mathrm{mM}) \\
\end{array}$ \\
\hline 1 & $\mathrm{NADH}+$ methylene blue + EDTA & + & - & - & + & - & + \\
\hline 2 & $\mathrm{NADH}+$ cytochrome $c+$ EDTA & + & - & - & - & + & + \\
\hline 3 & Ascorbate + methylene blue + EDTA & - & + & - & + & - & + \\
\hline 4 & Ascorbate + cytochrome $c+$ EDTA & - & - & + & - & + & + \\
\hline
\end{tabular}

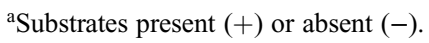

$\mathrm{EDTA}=$ ethylenediaminetetraacetic acid; $\mathrm{NADH}=$ nicotinamide adenine dinucleotide, reduced form.

microplate reader (San Jose, CA). Due to the delay in reduction at $4{ }^{\circ} \mathrm{C}$, the absorbance was measured for 90 min versus the $25 \mathrm{~min}$ used for the evaluation of the treatments at $25^{\circ} \mathrm{C}$. The reactions at $25^{\circ} \mathrm{C}$ were measured on the kinetic option on the Molecular Devices SpectraMax M3 Multi-mode microplate reader (San Jose, CA). The nonenzymatic MetMb and MetHb reductions for all treatments were determined during the linear phase of the reaction. A cofactor/electron donor combination with greatest $\mathrm{MetMb}$ reduction is presented in Figure 1 to demonstrate the spectral changes from 450 to $700 \mathrm{~nm}$ during 0,25 , and $90 \mathrm{~min}$ of incubation.

\section{Experiment 3: Evaluation of the $\mathrm{pH}$ effect on cofactors}

The assay mixture was evaluated in phosphate buffers $(50 \mathrm{mM})$ at $\mathrm{pH}$ of 5.2, 5.6, 6.0, and 6.4 to determine the effect of $\mathrm{pH}$ on cofactors at room temperature (Table 2). As indicated in experiment 2, a greater concentration of ascorbate was utilized to enhance MetMb reduction in presence of cyt-c. The absorbance was measured for all treatments for $25 \mathrm{~min}$ to calculate nonenzymatic MetMb and MetHb reduction.

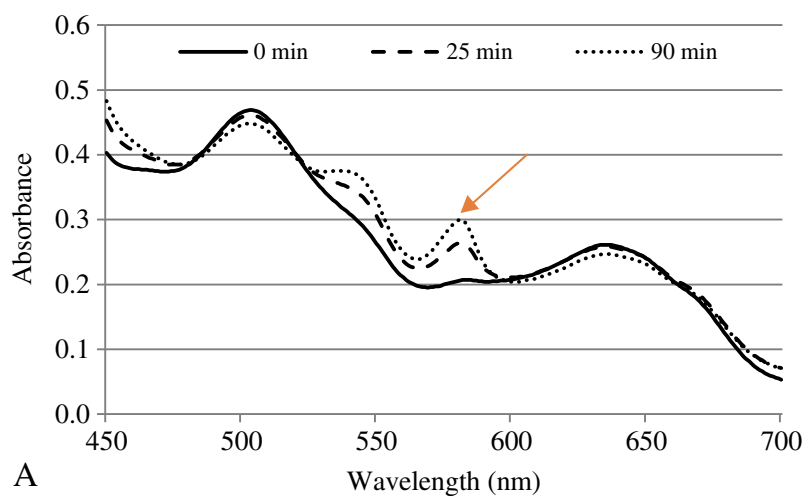

\section{Statistical analysis}

The experimental design was a completely randomized design. The experiments 1 and 2 were replicated 6 times $(n=6)$, and experiment 3 was replicated 5 times $(n=5)$. For experiment 1 , the fixed effects were substrate combinations, $\mathrm{pH}$, and temperature. For experiment 2 and 3, substrates combination was considered a fixed effect. Due to large differences in nonenzymatic MetMb and MetHb reduction among treatments and variance heterogeneity, nanomoles of $\mathrm{MetMb}$ and $\mathrm{MetHb}$ reduction were transformed to logarithm of nanomoles of MetMb and MetHb reduction before analysis. The MIXED procedure of SAS version 9.4 (SAS Institute Inc., Cary, NC) was used for all objectives to determine the Type 3 tests of fixed effects. The model statement included substrate combinations (experiment 1 ), a combination of temperature $\times$ substrate combinations (experiment 2 ), and a combination of substrate combinations $\times \mathrm{pH}$ (experiment 3 ). Least square means were considered significant at $P<0.05$ and were separated using the PDIFF option $(P<0.05)$. The standard error for treatments were reported for all objectives. The data for MetMb and $\mathrm{MetHb}$ reduction were analyzed separately to avoid

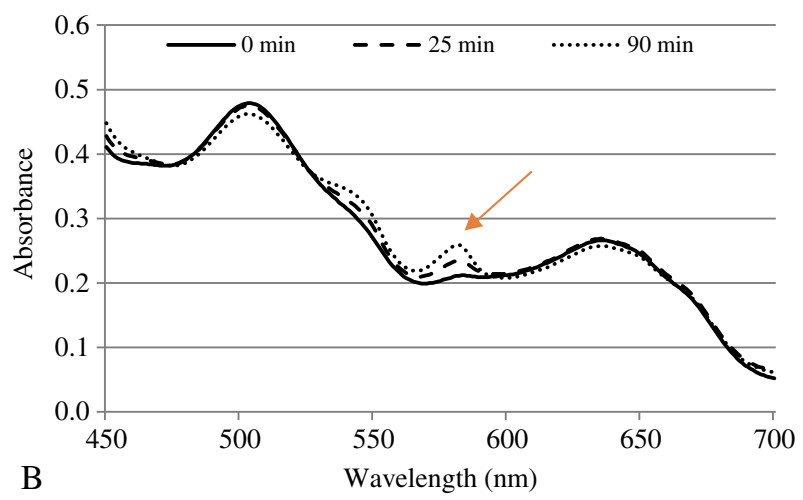

Figure 1. Spectral data from 450 to $700 \mathrm{~nm}$ at 0,25 , and $90 \mathrm{~min}$ for nonenzymatic reduction of metmyoglobin in the presence of NADH + methylene blue + EDTA (panel A) and ascorbate + methylene blue + EDTA (panel B) at pH 5.6 and $4^{\circ} \mathrm{C}$. The arrow indicates the wavelength maxima for oxymyoglobin $(582 \mathrm{~nm})$. EDTA, ethylenediaminetetraacetic acid; NADH, nicotinamide adenine dinucleotide, reduced form 
the complexity of the number of treatments. However, least square means for MetMb and MetHb reduction were compared numerically to understand the changes.

\section{Results}

\section{Effects of cofactors on nonenzymatic MetMb and MetHb reduction}

There was a significant cofactor effect on nonenzymatic MetMb reduction (Figure 2A). Nonenzymatic MetMb reduction was greatest in treatments containing NADH plus methylene blue, followed by ascorbate and cyt-c, and NADH combined with cyt-c or alone. More nonenzymatic MetMb reduction occurred in treatments with methylene blue than treatments with cyt-c $(P<0.05)$. Addition of methylene blue to NADH increased $(P<0.0001)$ nonenzymatic MetMb reduction compared with NADH alone, but the addition of EDTA to methylene blue and NADH did not impact $(P=0.91)$ nonenzymatic MetMb reduction. Ascorbate paired with cyt-c had greater $(P=0.02)$ nonenzymatic MetMb reduction than NADH plus cyt-c.

Nonenzymatic MetHb reduction was also affected by combinations of different cofactors and electron donors $(P<0.05$; Figure 2B). Treatment with $\mathrm{NADH}$ alone resulted in the lowest amounts of $\mathrm{MetHb}$ reduction compared with all other cofactor combinations. The addition of methylene blue to NADH caused an increase $(P<0.0001)$ in nonenzymatic MetHb reduction. However, a lower MetHb reduction $(P<0.0001)$ was observed when cyt-c added as an electron carrier instead of methylene blue with NADH. Furthermore, replacing NADH with ascorbate as the electron donor paired with cyt-c resulted in greater $(P<0.0001)$ MetHb reduction than NADH combined with cyt-c. The presence or absence of EDTA showed no changes $(P=0.38)$ in nonenzymatic MetHb reduction when combined with NADH and methylene blue. MetHb reduction for the NADH/ methylene blue and NADH/cyt-c electron donor/ carrier pair was numerically lower than the MetMb reduction. NADH without an electron carrier reduced more MetMb than MetHb.

\section{Effects of temperature on nonenzymatic MetMb and MetHb reduction}

Temperature did not have an effect on nonenzymatic MetMb reduction with NADH plus cytochrome $c$ plus EDTA but had an impact $(P<0.05)$ for ascorbate plus cytochrome $c$ plus EDTA, for ascorbate plus methylene blue plus EDTA, and for NADH plus methylene blue plus EDTA (Figure 3A). With increasing temperature from $4^{\circ} \mathrm{C}$ to $25^{\circ} \mathrm{C}$, treatments containing methylene blue had lower MetMb reduction. Ascorbate in combination with cyt-c increased $(P=$ $0.007)$ nonenzymatic MetMb reduction with increasing temperature from $4^{\circ} \mathrm{C}$ to $25^{\circ} \mathrm{C}$.

Decreasing the reaction temperature from $25^{\circ} \mathrm{C}$ to $4^{\circ} \mathrm{C}$ resulted in greater $(P=0.003) \mathrm{MetHb}$ reduction by NADH/cyt-c electron donor/carrier pair (Figure 3B). However, when the electron donor was replaced with ascorbate, decreasing the temperature
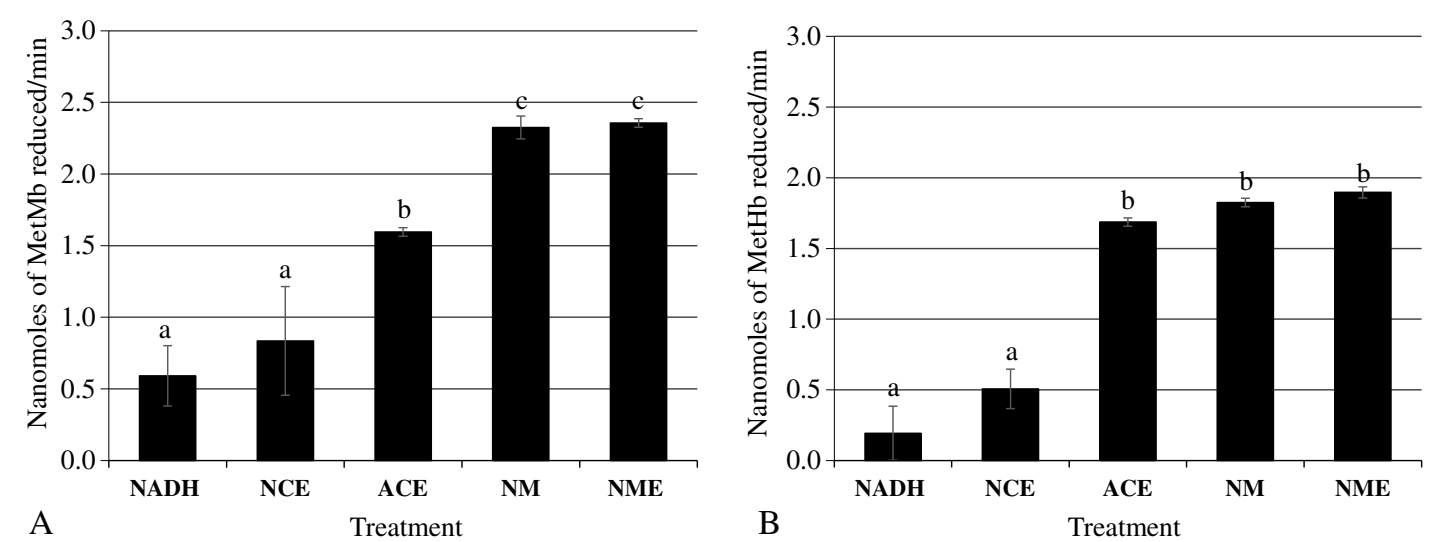

Figure 2. Least square means for cofactor treatments evaluating the effects of NADH + cytochrome $c+$ EDTA (NCE), ascorbate + cytochrome $c+$ EDTA (ACE), NADH + methylene blue (NM), and NADH + methylene blue + EDTA (NME) with equine metmyoglobin (A; 0.08 mM) and bovine hemoglobin $(\mathrm{B} ; 0.08 \mathrm{mM})$ on nonenzymatic metmyoglobin and methemoglobin reduction at $25^{\circ} \mathrm{C}$ and $\mathrm{pH} 5.6$. Least square means with different letters $(\mathrm{a}-\mathrm{c})$ are significantly different within panel $\mathrm{A}$, and least square means with different letters $(\mathrm{a}-\mathrm{b})$ are significantly different within panel $\mathrm{B}(P<0.05)$. Error bars indicate standard error of the treatment. EDTA, ethylenediaminetetraacetic acid; NADH, nicotinamide adenine dinucleotide, reduced form 

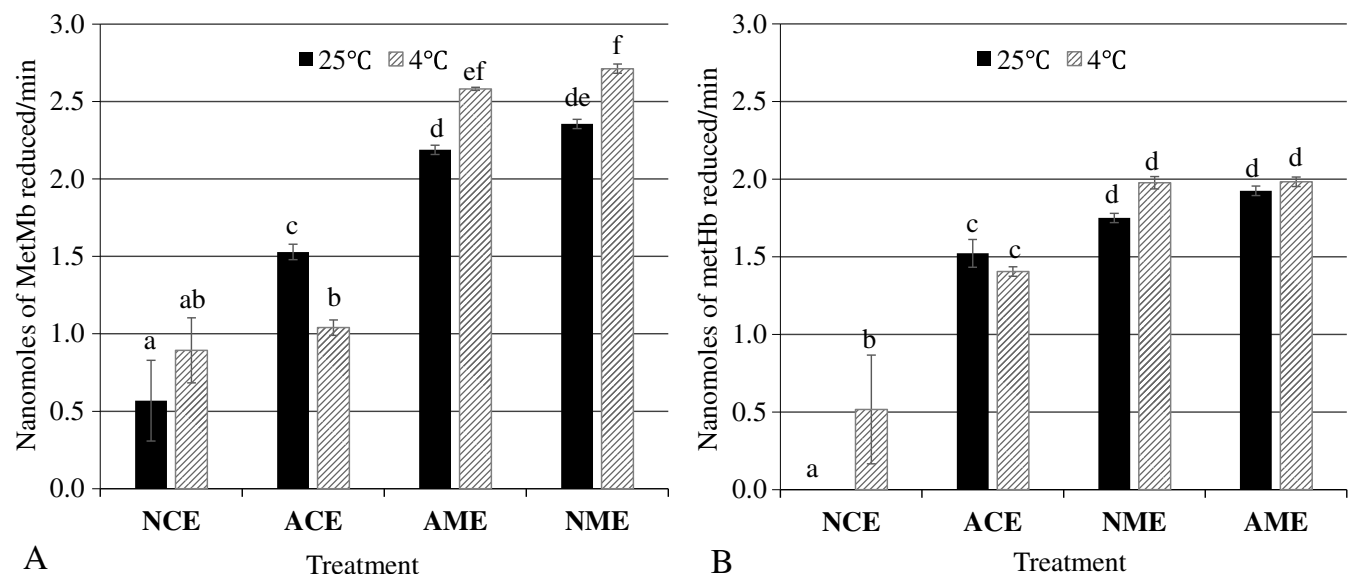

Figure 3. Least square means for cofactor treatments of NADH + cytochrome $c+$ EDTA (NCE), ascorbate + cytochrome $c+$ EDTA (ACE), ascorbate + methylene blue + EDTA (AME), and NADH + methylene blue + EDTA (NME) on nonenzymatic metmyoglobin (panel A) and methemoglobin (panel B) reduction at $4^{\circ} \mathrm{C}$ and $25^{\circ} \mathrm{C}$ and $\mathrm{pH} 5.6$. Least square means with different letters (a-f) are significantly different within panel A, and least square means with different letters $(\mathrm{a}-\mathrm{d})$ are significantly different within panel $\mathrm{B}(P<0.05)$. Error bars indicate standard error of the treatment. EDTA, ethylenediaminetetraacetic acid; $\mathrm{NADH}$, nicotinamide adenine dinucleotide, reduced form

did not result $(P=0.46)$ in greater levels of nonenzymatic MetHb reduction. Furthermore, when methylene blue was used as an electron carrier, the temperature of the reaction had no significant effect on the amount of MetHb reduced regardless of electron donor. Overall, all other cofactor combinations showed a greater rate of nonenzymatic MetHb reduction $(P<0.05)$ than the NADH/cyt-c electron donor/ carrier pair. Numerically, nonenzymatic reduction of $\mathrm{MetHb}$ was lower than reduction of MetMb for $\mathrm{NADH} /$ methylene blue electron donor/carrier pair at both temperatures. Reduction of MetMb was numerically lower at $4{ }^{\circ} \mathrm{C}$ than reduction of MetHb in the presence of ascorbate and cyt-c.

\section{Effects of $p H$ on nonenzymatic MetMb and Met $H b$ reduction}

Evaluation of nonenzymatic MetMb reduction at $\mathrm{pH} 5.2$ to 6.4 revealed a significant $\mathrm{pH}$ effect on the nonenzymatic MetMb reduction (Figure 4A). At $\mathrm{pH}$ 6.0 and 6.4, the nonenzymatic MetMb reduction for all electron donor/carrier pairs were not different $(P>$ 0.05). As $\mathrm{pH}$ increased, MetMb reduction decreased for methylene blue paired with NADH. The nonenzymatic MetMb reduction of the $\mathrm{NADH} /$ methylene blue electron donor/carrier pair was greater $(P<0.05)$ than all other treatments at $\mathrm{pH}$ lower than 5.6. cyt-c combined with NADH increased $(P<0.05)$ MetMb
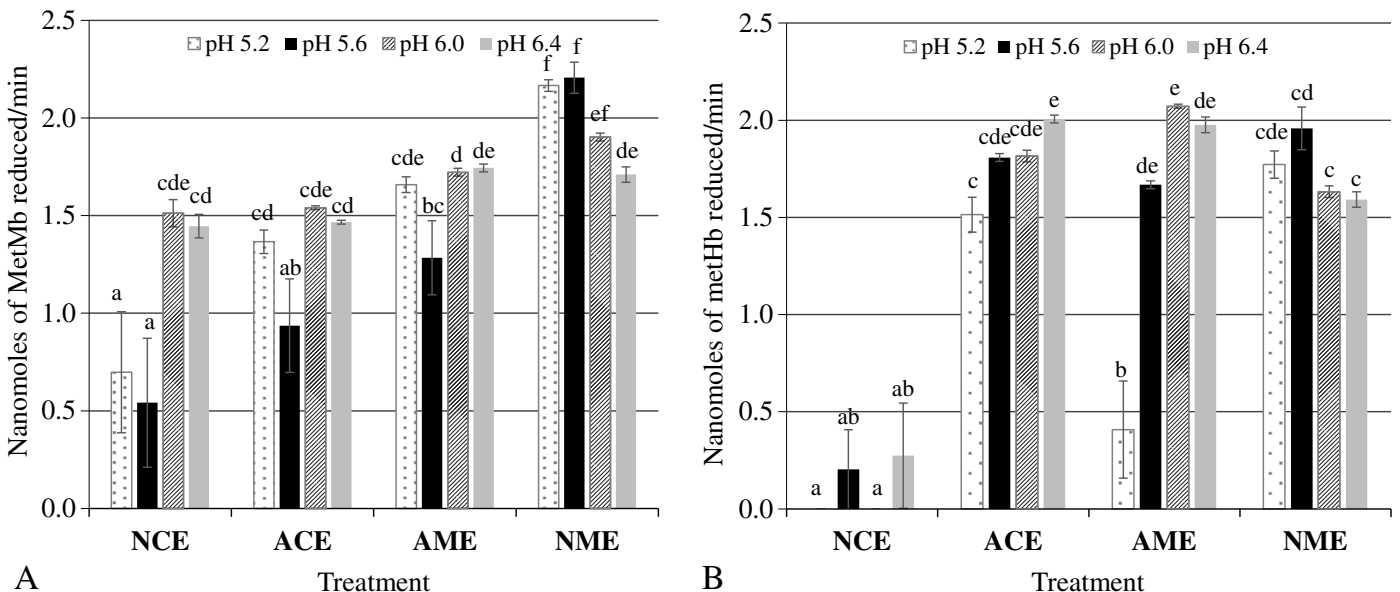

Figure 4. Least square means for cofactor treatments of NADH + cytochrome $c+$ EDTA (NCE), ascorbate + cytochrome $c+$ EDTA (ACE), ascorbate + methylene blue + EDTA (AME), and NADH + methylene blue + EDTA (NME) on the nonenzymatic metmyoglobin (panel A) and methemoglobin (panel B) reducing ability of the cofactors from $\mathrm{pH} 5.2$ to 6.4 at $25^{\circ} \mathrm{C}$. Least square means with different letters (a-f) are significantly different within panel $\mathrm{A}$, and least square means with different letters $(\mathrm{a}-\mathrm{e})$ are significantly different within panel $\mathrm{B}(P<0.05)$. Error bars indicate standard error of the treatment. EDTA, ethylenediaminetetraacetic acid; NADH, nicotinamide adenine dinucleotide 
reduction at a $\mathrm{pH}$ greater than 6.0 . When pairing both electron carriers with ascorbate, nonenzymatic MetMb reduction was lowest at $\mathrm{pH}$ 5.6. Overall, changes in $\mathrm{pH}$ did not lead to a discernible trend in the nonenzymatic $\mathrm{MetMb}$ reduction of treatments with ascorbate (except at $\mathrm{pH}$ 5.6).

Altering reaction $\mathrm{pH}$ showed significant changes in nonenzymatic $\mathrm{MetHb}$ reduction among the various cofactors except for the electron donor/carrier pair combination of NADH and cyt-c (Figure 4B). Nonenzymatic MetHb reduction was greater $(P=0.004)$ at $\mathrm{pH} 6.4$ than 5.2 for ascorbate and cyt-c combination. When utilizing $\mathrm{NADH}$ and methylene blue, MetHb reduction was not impacted by $\mathrm{pH}$. There was no change in $\mathrm{MetHb}$ reduction as the $\mathrm{pH}$ increased from 5.6 to $6.0(P=0.48)$ and $6.4(P=0.91)$ for the ascorbate/methylene blue electron donor/carrier pair. However, when ascorbate/methylene blue reaction took place at $\mathrm{pH} 5.2$, nonenzymatic $\mathrm{MetHb}$ reduction was lower $(P<0.0001)$ than when the reaction took place at higher $\mathrm{pH}$ levels.

Numerically comparing the reduction of MetHb to $\mathrm{MetMb}, \mathrm{NADH} /$ cyt-c electron donor/carrier reduced MetMb more than MetHb at all $\mathrm{pH}$ levels except 5.6. Ascorbate paired with cyt-c resulted in more MetHb reduction than $\mathrm{MetMb}$ reduction at reaction $\mathrm{pH}$ of 5.6 to 6.4. Less reduction of MetHb occurred in the presence of methylene blue and NADH at lower $\mathrm{pH}$ compared to reduction of MetMb. However, numerically more reduction of MetHb than MetMb reduction happened at all $\mathrm{pH}$ levels except 5.2 for the ascorbate/ methylene blue electron donor/carrier pair.

\section{Discussion}

The ferric form of myoglobin and hemoglobin is inactive, therefore physiologically, reduction pathways are present to limit oxidized form accumulation in tissue. However, limited research has determined the effects of electron donors/carriers on nonenzymatic MetMb and $\mathrm{MetHb}$ reduction at postmortem muscle conditions. Brown and Snyder (1969) demonstrated nonenzymatic reduction of MetMb in the presence of NADH and flavin mononucleotide. Nevertheless, limited MetMb/MetHb reduction was observed with NADH alone (Koizumi and Brown, 1972; Hagler et al., 1979; Mikkelsen and Skibsted, 1992). Aligning with the results of the present study, nonenzymatic MetHb reduction was minimal by NADH without electron carriers.

Previous research on nonenzymatic reduction of $\mathrm{MetMb}$ indicated that the presence of EDTA is necessary for reduction by NADH (Brown and Snyder,
1969). However, no differences in nonenzymatic reduction were noticed between NADH plus methylene blue and NADH plus methylene blue plus EDTA. Similar results for EDTA effectiveness were noted in nitrosyl-MetMb reduction to nitric oxide myoglobin by various reducing agents (Fox Jr. and Ackerman, 1968). Pascal and Tarbell (1957) indicated that EDTA prevented metal catalysis in $\mathrm{pH}$-dependent reaction.

Brown and Snyder (1969) reported that methylene blue increased nonenzymatic reduction in the presence of NADH. In support, a greater nonenzymatic MetMb and $\mathrm{MetHb}$ reduction was observed in this study. Methylene blue is a redox agent with mild redox potential allowing it to cycle from oxidized and reduced forms (Atamna et al., 2012), and this allows methylene blue to act as an electron carrier in the reduction of $\mathrm{MetMb}$ and MetHb. Past research on other electron carriers such as cyt-c paired with NADH has focused on the redox reactions in the mitochondria and not on the nonenzymatic MetMb/MetHb reactions (Bernardi and Azzone, 1981; Lofrumento et al., 1991). Mitochondria undergo degenerative changes with increased postmortem time and can release cyt-c to sarcoplasm. Presence of cyt-c in sarcoplasm is an indicator of mitochondrial degeneration and initiation of apoptosis (Ke et al., 2017). Therefore, cyt-c in sarcoplasm has the potential to initiate nonenzymatic MetMb reduction.

Ascorbate can reduce MetMb in presence of ferrocyanide (Hagler et al., 1979; Mikkelsen and Skibsted, 1992). Ascorbate has been shown to nonenzymatically reduce $\mathrm{MetHb}$ at concentrations ranging from 6 to $18 \mathrm{mM}$ in aerobic and anaerobic conditions (Vestling, 1942; Tomoda et al., 1978a; Tomoda et al., 1978b; Sullivan and Stern, 1982; Dötsch et al., 1998); however, the reduction occurred more quickly in the presence of methylene blue (Vestling, 1942). In the current research, a combination of ascorbate and methylene blue resulted in a rapid reduction of $\mathrm{MetHb}$ and $\mathrm{MetMb}$. A previous study demonstrated that a greater concentration of ascorbate (130 to $1,500 \mathrm{mM})$ was required to nonenzymatically reduced MetMb because of the largeness of ascorbate making direct reduction of the heme difficult (Tsukahara and Yamamoto, 1983). The size of ascorbate may be attributed to the need for an electron carrier such as methylene blue to carry electron to heme at lower concentrations of ascorbate.

In the present study, the introduction of both electron carriers resulted in MetMb and MetHb reduction by ascorbate. The pKa of ascorbic acid is $4.7(\mathrm{pKa}$ represents the negative log of the acid dissociation constant). Since the $\mathrm{pH}$ of the current reaction was greater 
than the pKa of ascorbic acid, the ascorbate will be in ionized form between $\mathrm{pH} 5.2$ and 6.4. Therefore, the $\mathrm{pH}$ had less impact on the reduction of MetMb with ascorbate as an electron donor, except at $\mathrm{pH}$ 5.6. The potential reason for lower nonenzymatic MetMb reduction at $\mathrm{pH} 5.6$ is not clear. We speculate that the net charge of myoglobin and charge of ascorbate at $\mathrm{pH}$ 5.6 may have limited electron transfer. However, in the current study, the reduction of MetHb increased at higher $\mathrm{pH}$ in the presence of ascorbate. Previous research indicated that ascorbate is an effective reducing agent of ferricytochrome $c$ (Schejter and Aviram, 1969; Myer et al., 1980). The mechanism proposed by Myer et al. (1980) noted that ascorbate binds to arginine-38 on cyt-c with electron movement through hydrogen bonds, tryptophan-59, or inner propionate side chain to the heme. The results from the current study support this mechanism of ascorbate oxidation to form a reduced cyt-c, which can act as an electron donor for MetMb or MetHb.

In the present research, cyt-c was not as effective in reducing MetMb compared with methylene blue. Past research showed that ferricytochrome $c$ in the presence of myoglobin led to formation of MetMb and ferrocytochrome $c$; however, no further reaction occurred once MetMb was formed, indicating that oxidation of ferrocytohemochrome $c$ to form oxymyoglobin was unlikely (Wu et al., 1972). More specifically, cyt-c contains a greater number of aromatic and aliphatic amino acids surrounding the heme, creating a hydrophobic environment. This environment increases the difficulty of the heme gaining and holding a positive charge in an oxidized state. The difficulty for cyt-c to be oxidized could explain the decrease in MetMb reduction in treatments containing cyt-c compared to those containing methylene blue as an electron carrier. In the present study, MetMb reduction increased at a higher $\mathrm{pH}$ in the presence of cyt-c and NADH. The redox potential of cyt-c can be affected by $\mathrm{pH}$. As $\mathrm{pH}$ is increased, the redox potential decreases, indicating that cyt-c is less likely to be reduced (Rodkey and Ball, 1950; Salemme, 1977). This is because the oxidized state of cyt-c is stabilized as the protonation of tyrosine decreases with greater $\mathrm{pH}$ (Salemme, 1977). Therefore, shifting the $\mathrm{pH}$ can affect the reducing ability of cyt-c.

Myoglobin oxidation occurs more readily at $25^{\circ} \mathrm{C}$ compared to $4^{\circ} \mathrm{C}$. Past research supports increased oxidation at higher temperatures (Snyder and Ayres, 1961; Brown and Mebine, 1969; Yin and Faustman, 1993). Oxidation of hemoglobin is more likely to occur at room temperature compared to $15^{\circ} \mathrm{C}$ (Brooks, 1931), supporting the lower amount of reduction at $25^{\circ} \mathrm{C} \mathrm{com}-$ pared to $4^{\circ} \mathrm{C}$ in this study. The results indicate that nonenzymatic MetMb and MetHb reductions are possible at postmortem muscle $\mathrm{pH}$ and lower temperature. At a lower temperature, more reaction time is required to have observed MetMb and MetHb reduction. Thus, incubation time ranged from 25 to 90 .

To the best of our knowledge, current research is the first to determine the effect of various cofactors on nonenzymatic MetMb and MetHb reduction at postmortem muscle and storage conditions. We are not aware of a methodology that will selectively inhibit mitochondrial and enzymatic MetMb and $\mathrm{MetHb}$ reduction to determine the relative contribution of nonenzymatic MetMb or MetHb reduction in postmortem muscle conditions. Although we did not compare $\mathrm{MetMb}$ and MetHb, differences in the reduction potential of cofactors and MetMb/MetMb may explain the differences in nonenzymatic MetMb and MetHb reduction. For example, the reduction potential properties of MetMb at $\mathrm{pH}$ 5.6, 6.4, and 7.4 were different (Nerimetla et al., 2014). A greater $\mathrm{pH}$ resulted in lower reduction potential and has a greater tendency for reduction (Nerimetla et al., 2017). Furthermore, changes in the reduction potential of electron donors can also explain $\mathrm{pH}$ - and temperature-dependent effects on MetMb and MetHb reduction differences.

\section{Conclusions}

The type of cofactor can impact nonenzymatic $\mathrm{MetMb}$ and MetHb reduction. Methylene blue is an effective electron carrier compared with cyt-c when combined with NADH and ascorbate in reducing MetMb. Temperature and $\mathrm{pH}$ effects on nonenzymatic $\mathrm{MetMb}$ and MetMb reduction are dependent on the specific cofactors. In contrast with myoglobin, methylene blue was not an effective electron carrier compared with cyt-c for MetHb reduction. The combination of ascorbate and cyt-c showed similar rates of nonenzymatic MetHb reduction compared to NADH and methylene blue. $\mathrm{pH}$ and temperature had limited impact on the rate of nonenzymatic MetHb reduction compared with various cofactors. Further, current research demonstrates that naturally present electron donor (ascorbate) and electron carrier (cyt-c) can result in nonenzymatic reduction at meat $\mathrm{pH}$ and storage conditions. The present research indicates that characterizing the cofactors that contribute to nonenzymatic $\mathrm{MetMb}$ and MetHb reduction has the potential to be utilized in post-harvest injection enhancement and/or 
active packaging technology to minimize losses due to discoloration.

\section{Literature Cited}

AMSA. 2012. Meat color measurement guidelines. Am. Meat Sci. Assoc., Champaign, IL.

Arihara, K., R. G. Cassens, M. L. Greaser, J. B. Luchansky, and P. E. Mozdziak. 1995. Localization of metmyoglobinreducing enzyme (NADH-cytochrome b5 reductase) system components in bovine skeletal muscle. Meat Sci. 39:205213. https://doi.org/10.1016/0309-1740(94)P1821-C.

Atamna, H., J. Mackey, and J. M. Dhahbi. 2012. Mitochondrial pharmacology: electron transport chain bypass as strategies to treat mitochondrial dysfunction. BioFactors. 38:158-166. https://doi.org/10.1002/biof.197.

Bernardi, P., and G. F. Azzone. 1981. Cytochrome c as an electron shuttle between the outer and inner mitochondrial membranes. J. Biol. Chem. 256:7187-7192.

Beutler, E., and M. C. Baluda. 1963. Methemoglobin reduction. Blood. 22:323-333.

Bowen, W. J. 1949. The absorption spectra and extinction coefficients of myoglobin. J. Biol. Chem. 179:235-245.

Brooks, J. 1931. The oxidation of haemoglobin to methaemoglobin by oxygen. P. R. Soc. B. 109:35-50.

Broumand, H., C. O. Ball, and E. F. Stier. 1958. Factors affecting the quality of prepackaged meat. II. E. Determining the proportions of heme derivatives in fresh meat. Food Technol. 12:65-77.

Brown, W. D., and L. B. Mebine. 1969. Autoxidation of oxymyoglobins. J. Biol Chem. 244:6696-6701.

Brown, W. D., and H. E. Snyder. 1969. Nonenzymatic reduction and oxidation of myoglobin and hemoglobin by nicotinamide adenine dinucleotides and flavins. J. Biol. Chem. 244:67026706.

Carpenter, C. E., D. P. Cornforth, and D. Whittier. 2001. Consumer preferences for beef color and packaging did not affect eating satisfaction. Meat Sci. 57:359-363. https://doi.org/10.1016/ S0309-1740(00)00111-X.

Dötsch, J., S. Demirakça, A. Cryer, J. Hänze, P. G. Kühl, and W. Rascher. 1998. Reduction of NO-induced methemoglobinemia requires extremely high doses of ascorbic acid in vitro. Intens. Care Med. 24:612-615. https://doi.org/10.1007/ s001340050623.

Elroy, N. N., J. Rogers, G. G. Mafi, D. L. VanOverbeke, S. D. Hartson, and R. Ramanathan. 2015. Species-specific effects on non-enzymatic metmyoglobin reduction in vitro. Meat Sci. 105:108-113. https://doi.org/10.1016/j.meatsci.2015. 03.010

Faustman, C., and R. G. Cassens. 1990. The biochemical basis for discoloration in fresh meat: a review. J. Muscle Foods. 1:217243. https://doi.org/10.1111/j.1745-4573.1990.tb00366.x.

Fox Jr., J. B., and S. A. Ackerman. 1968. Formation of nitric oxide myoglobin: mechanisms of the reaction with various reductants. J. Food Sci. 33:364-370. https://doi.org/10.1111/j. 1365-2621.1968.tb03631.x.
Grunwald, E. W., and M. P. Richards. 2006. Mechanisms of heme protein-mediated lipid oxidation using hemoglobin and myoglobin variants in raw and heated washed muscle. J. Agr. Food Chem. 54:8271-8280. https://doi.org/10.1021/ jf061231d.

Hagler, L., R. I. Coppes Jr., and R. H. Herman. 1979. Metmyoglobin reductase. Identification and purification of a reduced nicotinamide adenine dinucleotide-dependent enzyme from bovine heart which reduces metmyoglobin. J. Biol. Chem. 254:6505-6514.

Ke, Y., R. M. Mitacek, A. Abraham, G. G. Mafi, D. L. Vanoverbeke, U. DeSilva, and R. Ramanathan. 2017. Effects of muscle-specific oxidative stress on cytochrome c release and oxidation-reduction potential properties. J. Agr. Food Chem. 65:7749-7755. https://doi.org/10.1021/ acs.jafc. $7 \mathrm{~b} 01735$.

Kendrew, J. C., R. E. Dickerson, B. E. Strandberg, R. G. Hart, D. R. Davies, D. C. Phillips, and V. C. Shore. 1960. Structure of myoglobin: a three-dimensional Fourier synthesis at $2 \AA$. resolution. Nature. 185:422-427.

Kim, Y. H., M. C. Hunt, R. A. Mancini, M. Seyfert, T. M. Loughin, D. H. Kropf, and J. S. Smith. 2006. Mechanism for lactatecolor stabilization in injection-enhanced beef. J. Agr. Food Chem. 54:7856-7862. https://doi.org/10.1021/jf061225h.

Koizumi, C., and W. D. Brown. 1972. A peroxidative mechanism for the nonenzymatic reduction of metmyoglobin. BBA-Gen. Subjects 264:17-24. https://doi.org/10.1016/0304-4165(72) 90112-2.

Liu, C., Y. Zhang, X. Yang, R. Liang, Y. Mao, X. Hou, X. Lu, and X. Luo. 2014. Potential mechanisms of carbon monoxide and high oxygen packaging in maintaining color stability of different bovine muscles. Meat Sci. 97:189-196. https://doi.org/10. 1016/j.meatsci.2014.01.027.

Lofrumento, N. E., D. Marzulli, L. Cafagno, G. La Piana, and T. Cipriani. 1991. Oxidation and reduction of exogenous cytochrome $\mathrm{c}$ by the activity of the respiratory chain. Arch. Biochem. Biophys. 288:293-301. https://doi.org/10.1016/ 0003-9861(91)90198-R.

Mikkelsen, A., and L. H. Skibsted. 1992. Kinetics of enzymatic reduction of metmyoglobin in relation to oxygen activation in meat products. Z. Lebensm. Unters. For. 194:9-16. https://doi.org/10.1007/BF01191032.

Mitacek, R. M., Y. Ke, J. E. Prenni, R. Jadeja, D. L. VanOverbeke, G. G. Mafi, and R. Ramanathan. 2019. Mitochondrial degeneration, depletion of $\mathrm{NADH}$, and oxidative stress decrease color stability of wet-aged beef longissimus steaks. J. Food Sci. 84:38-50. https://doi.org/10.1111/1750-3841.14396.

Myer, Y. P., K. K. Thallam, and A. Pande. 1980. Kinetics of the reduction of horse heart ferricytochrome c. ascorbate reduction in the presence and absence of urea. J. Biol Chem. 255:9666-9673.

Nerimetla, R., C. Walgama, R. Ramanathan, and S. Krishnan. 2014. Correlating the electrochemical kinetics of myoglobinfilms to pH dependent meat color. Electroanal. 26:675-678. https:// doi.org/10.1002/elan.201300630.

Nerimetla, R., S. Krishnan, S. Mazumder, S. Mohanty, G. G. Mafi, D. L. VanOverbeke, and R. Ramanathan. 2017. Speciesspecificity in myoglobin oxygenation and reduction potential 
properties. Meat Muscle Biol. 1:1-7. https://doi.org/10. 22175/mmb2016.10.0003.

Pascal, I., and D. S. Tarbell. 1957. The kinetics of the oxidation of a mercaptan to the corresponding disulfide by aqueous hydrogen peroxide. J. Am. Chem. Soc. 79:6015-6020. https://doi.org/10.1021/ja01579a045.

Perutz, M., M. G. Rossmann, A. F. Cullis, H. Muirhead, G. Will, and A. C. T. North. 1960. Structure of haemoglobin: A threedimensional Fourier synthesis at 5.5-A. resolution, obtained by X-ray analysis. Nature. 185:416-422. https://doi.org/10. 1038/185416a0

Ramanathan, R., and R. A. Mancini. 2018. Role of mitochondria in beef color: a review. Meat Muscle Biol. 2:309-320. https:// doi.org/10.22175/mmb2018.05.0013.

Richards, M. P., A. M. Modra, and R. Li. 2002. Role of deoxyhemoglobin in lipid oxidation of washed cod muscle mediated by trout, poultry and beef hemoglobins. Meat Sci. 62:157-163. https://doi.org/10.1016/S0309-1740(01) 00242-X.

Rodkey, F. L., and E. G. Ball. 1950. Oxidation-reduction potentials of the cytochrome c system J. Biol. Chem. 182:17-28.

Salemme, F. R. 1977. Structure and function of cytochromes c. Annu. Rev. Biochem. 46:299-330. https://doi.org/10.1146/ annurev.bi.46.070177.001503.

Schejter, A., and I. Aviram. 1969. Reaction of cytochrome c with imidazole. Biochemistry-US 8:149-153. https://doi.org/10. 1021/bi00829a021.

Snyder, H. E., and J. C. Ayres. 1961. The autoxidation of crystallized beef myoglobin. J. Food Sci. 26:469-474. https://doi. org/10.1111/j.1365-2621.1961.tb00391.x.

Sullivan, S. G., and A. Stern. 1982. Effects of ascorbate on methemoglobin reduction in intact red cells. Arch. Biochem. Biophys. 213:590-594. https://doi.org/10.1016/0003-9861 (82)90588-4
Tang, J., C. Faustman, R. A. Mancini, M. Seyfert, and M. C. Hunt. 2005. Mitochondrial reduction of metmyoglobin: dependence on the electron transport chain. J. Agr. Food Chem. 53:5449 5455. https://doi.org/10.1021/jf050092h.

Tomoda, A., M. Takeshita, and Y. Yoneyama. 1978a. Characterization of intermediate hemoglobin produced during methemoglobin reduction by ascorbic acid. J. Biol. Chem. 253:7415-7419.

Tomoda, A., A. Tsuji, S. Matsukawa, M. Takeshita, and Y. Yoneyama. 1978b. Mechanism of methemoglobin reduction by ascorbic acid under anaerobic conditions. J Biol. Chem. 253:7240-7243.

Tsukahara, K., and Y. Yamamoto. 1983. Kinetic studies on the reduction of metmyoglobins by ascorbic acid. J. Biochem. 93:15-22. https://doi.org/10.1093/oxfordjournals.jbchem. a134149.

Vestling, C. S. 1942. The reduction of methemoglobin by ascorbic acid. J. Biol. Chem. 143:439-446.

Wu, C.-S. C., P. Duffy, and W. D. Brown. 1972. Interaction of myoglobin and cytochrome c. J. Biol. Chem. 247:18991903.

Yin, J., W. Zhang, and M. P. Richards. 2017. Attributes of lipid oxidation due to bovine myoglobin, hemoglobin and hemolysate. Food Chem. 234:230-235. https://doi.org/10.1016/j. foodchem.2017.04.182.

Yin, M. C., and C. Faustman. 1993. Influence of temperature, pH, and phospholipid composition upon the stability of myoglobin and phospholipid: a liposome model. J. Agr. Food Chem. 41:853-857. https://doi.org/10.1021/jf00030a002.

Zijlstra, W. G., and A. Buursma. 1997. Spectrophotometry of hemoglobin: absorption spectra of bovine oxyhemoglobin, deoxyhemoglobin, carboxyhemoglobin, and methemoglobin. Comp. Biochem. Phys. B. 118:743-749. https://doi.org/10. 1016/S0305-0491(97)00230-7. 